\title{
MICRO-FLUIDIC SYSTEM WITH GROOVED POLE PIECES OF MAGNETIC CIRCUIT
}

\author{
O. Macháček*, M. Kubík, J. Roupec, Z. Strecker, I. Mazůrek
}

\begin{abstract}
Micro-fluidic systems are used in approximately 30 years for detect or separate biological molecules which are contain in a biological medium (blood, serum, etc.). The technique of separation is based on magnetic force acting on the magnetic particles which are added to the medium. One way to increase separation efficiency is to optimize the magnetic circuit. Two versions of magnetic circuit for the micro-fluidic system are designed, tested and compared in this study. The first of them has solid pole pieces (without grooves), the second one is provided with grooves which are oriented perpendicular to the direction of a fluid flow through the system. A suitable geometry of the grooves was chosen using a FEM model which is verified by measurement. Hypothesis that the grooved pole pieces ensure higher amount of captured particles in the system in comparison with the case of solid pole pieces was confirmed. But, the comparison of the captured particles amount was made just visually. Therefore, the amount of particles which are washout during a flow test should be evaluated by more quantifiable method in future study, for example using Beer-Lambert law.
\end{abstract}

Keywords: micro-fluidic system, immunoassay, magnetic circuit, grooved pole pieces

\section{Introduction}

Magnetic immunoassay is one of multiple techniques of biological analysis using to confirm or disprove occurrence of an unexpected molecules in biological medium such as blood, serum, cell lysate etc. This assay is used for example for cancer diagnostics, sports anti-doping analysis etc., the aim is to detect or separate target biological molecules called antigens. This technique usually uses micro-size magnetic particles with captured antibodies on its surface. The target antigens are bound to the capture antibodies due to strong molecular interactions between them, Peng et al. (2011). The magnetic micro-beads with captured antigens can be easily separated from the medium by the application of magnetic field. The separation of magnetic particles is carried out in micro-fluidic systems.

The fluid with the magnetic particles flow through the micro-fluidic system, when the magnetic field is applied, the particles are captured in the system while non-magnetic medium is flowing away. The first microfluidic system was described by Manz et al. (1990). Since then, an efficiency of the microfluidic systems is improving. That means to solve two conflicting tasks. The first task is high sensitivity and the second one is low process time of the assay.

The sensitivity of the assay grows, when small particles are used, because there is high ratio between the surface and volume of the particles. Therefore, much more antigens can be captured in the same volume of the particles. On the other hand, the smaller particles are used the lower flow rate of the medium can be applied, because of weak magnetic forces. That means the time of assay process grows. One option how to achieve high sensitivity of the assay while the flow rate is quite high showed Kuzhir (2017), he used micro-pillars which were fixed in the chip to capture particles of mean diameter around $60 \mathrm{~nm}$.

Three most common problems of micro-fluidic system are insufficient fixation of magnetic particles, which limited the maximal flow rate or particle size, particle aggregation in the system which reduces

* All authors: Institute of Machine and Industrial Design, Brno University of Technology, Technická 2896/2, 61669 Brno; CZ, Ing. Ondřej Macháček: Ondrej.Machacek@vutbr.cz 
the sensitivity of the analysis, and finally complicated washing of the magnetic particles out of the system. There are several commercial micro-fluidic systems with various shapes of working chamber - a chip. The chip which is used in one of common micro-fluidic system, ChipGenie was used for example in study of Svobodova et al. (2015), is also used for the experiments which are described below.

The aim of this article is to design, manufacture and test a new magnetic circuit for the micro-fluidic system described above and try to improve fixation of magnetic particles and to simplify process of cleaning out compared to the ChipGenie. The magnetic circuit will be designed using FEM magnetostatic model this method is described a verified by Kubik et al. (2017).

\section{Methods}

The preliminary measurement showed that the particles were captured especially near inlet and outlet edges of magnetic circuit with no grooves. Grooved pole pieces of magnetic circuit in the perpendicular direction to the fluid flow should increase particles fixation. This hypothesis is based on study of Zou and $\mathrm{Lu}$ (1992), this study deals with magnetic seal. The FEM model of magnetic circuit was created to determine the grooves geometry which provides high differences between the maximum and minimum of magnetic flux density along the magnetic particles flow path in the chip. The flow test was provided with the same parameters for magnetic circuit with 3 grooves / with no grooves and the amount of captured particles was compared visually.

\subsection{FEM model of magnetic circuit}

The 3D model was created in software Ansys Electronics 2018, the geometry of magnetic circuit was simplified using symmetry relative to plane XY. The geometry of the magnetic circuit is shown in Fig. 1a. The boundary was set as a box outside any part of model. The excitation on the coil was magnetomotive force $I^{*} 600 A^{*}$ turns. Length based mesh (maximum $3 \mathrm{~mm}$ ) was applied in magnetic circuit, the material properties of this part were defined using measured B-H curve of pure iron called Behanit. The mesh was refined in the gap (max. length $0.4 \mathrm{~mm}$ ), see Fig. 1b. The material of elements in the gap was set as vacuum. The model helps to find a combination of dimensional parameters which provides high difference between maximum and minimum magnetic flux density along the line in the middle of the gap, the line is parallel to $\mathrm{X}$ axis.

a)

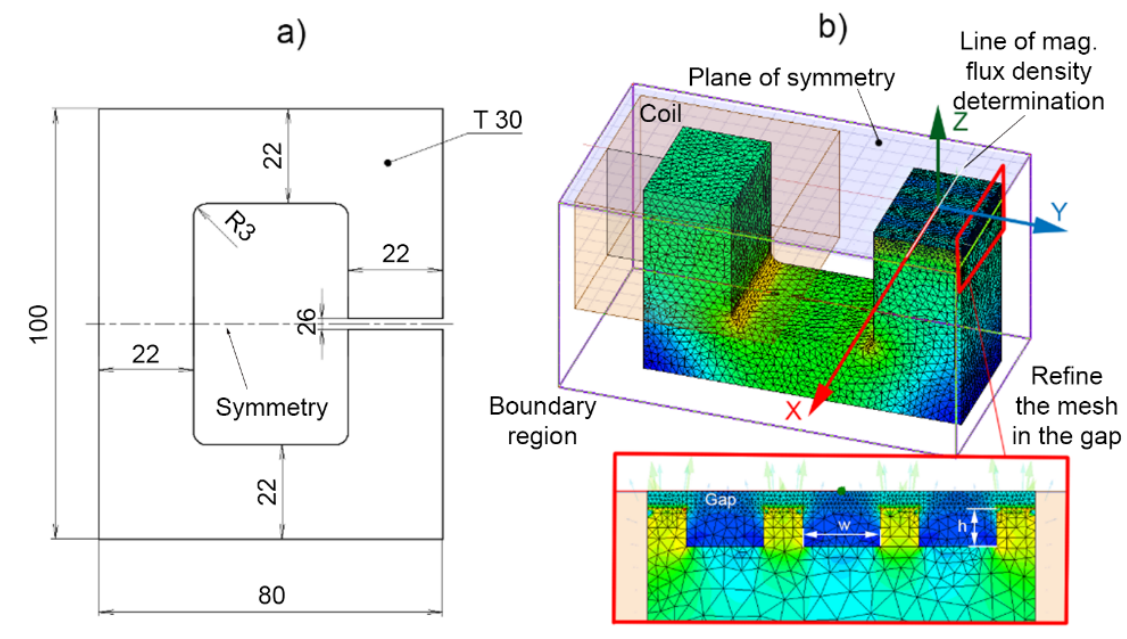

Fig. 1: FEM model of the micro-fluidic system magnetic circuit.

\subsection{Measurement set up}

Two measurements were provided in this study. The first was measurement of magnetic flux density along the gap of magnetic circuit; see Fig. 2a. The magnetic circuit was manufactured with the same dimensions as the simulation was performed in two versions. The coil was wound with 600 turns and it was excited by electric current using power supply Manson SDP2603. The magnetic flux density position dependency (B-x) in the middle of the gap was measured by standard hall probe STD 18-04-04 which was connected to magnetometer Bell and the position was measured by an optical sensor Baumer OADM 20U2472/S14C. The signals were analyzed in data acquisition system DEWE 800. 


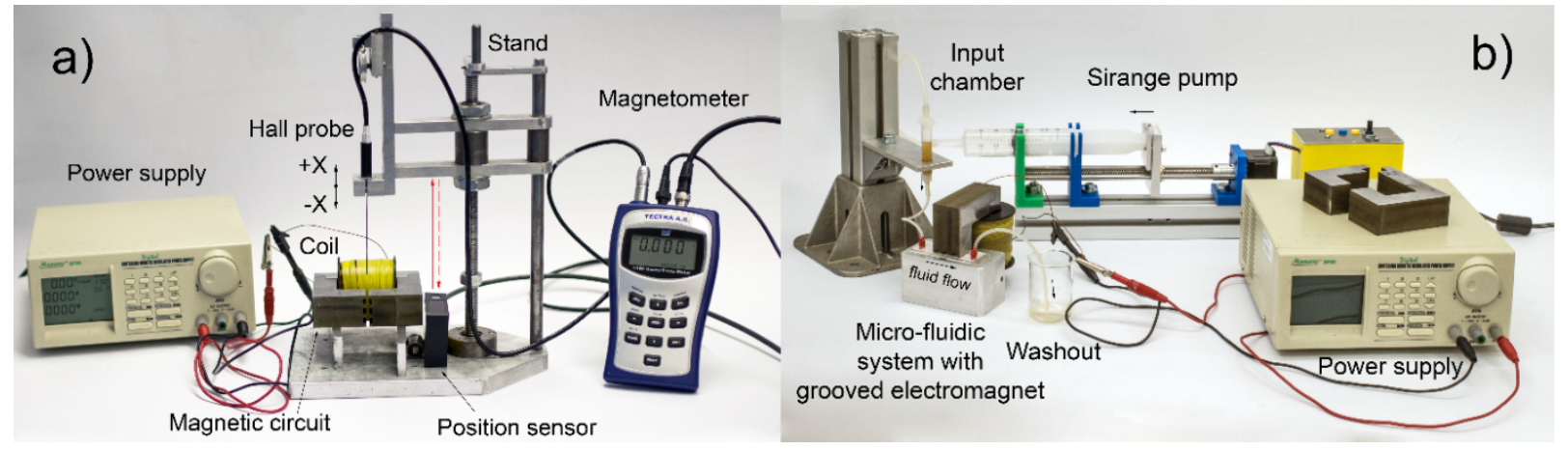

Fig. 2: Magnetic flux density measurement a) and the flow test b)

Secondly, a flow test of a fluid through the micro-fluidic system was provided, see Fig. $2 b$. The fluid is a suspension, which consist of $1 \mathrm{mg}$ of magnetic particles Seradyne with mean diameter $816 \mathrm{~nm}$ and $2 \mathrm{ml}$ of biofunctionalized carrier - buffer. Capturing efficiency of the micro-fluidic system with grooved or solid pole pieces of magnetic circuit was compared for the constant flow rate $5 \mathrm{ml} / \mathrm{min}$ by following procedure. At first, $2 \mathrm{ml}$ of the suspension flows from the input chamber into the chip of micro-fluidic system where the particles are captured by magnetic field which is generated by a coil powered by the current $I=2.5 \mathrm{~A}$. After this $10 \mathrm{ml}$ of buffer is filled in the input chamber and it is the forced to flow through the chip. The amount of washout particles is monitored. Then the current in the coil is decreased to $2 \mathrm{~A}, 2 \mathrm{ml}$ of buffer is forced to flow through the chip and the amount of washout particles is monitored again. Consequently, this method is re-performed for currents: $2 \mathrm{~A} ; 1.5 \mathrm{~A} ; 1.25 \mathrm{~A} ; 1 \mathrm{~A} ; 0.75 \mathrm{~A}$ and $0.5 \mathrm{~A}$. Finally, the current in the coil is set to $0 \mathrm{~A}, 10 \mathrm{ml}$ of buffer is used for washout the rest of particles from the chip.

\section{Results}

\subsection{Magnetic flux density in the gap}

Influence of three parameters of pole pieces to magnetic flux density in the gap was analyzed in the FEM model for the constant electric current $\mathrm{I}=2 \mathrm{~A}$, see Fig. $1 \mathrm{~b}$. The parameters were: number $(i)$, height $(h)$ and width $(w)$ of grooves. Magnetic flux density - position dependency (B-x) obtained by these analyses will be shown during the presentation. However, the analyses demonstrated several facts. Number of grooves has minimum impact to max. and min. magnetic flux density in the gap, see Fig. 3a. The width of grooves affects the value of minimum magnetic flux density in the gap almost linearly, the maximum of magnetic flux density in the gap increases very little for wide grooves. The ratio $R$ between min. and max. magnetic flux density $\left(R=B_{\min } / B_{\max }\right)$ is plotted in Y axes of Fig. 3a. The height of grooves affects the minimum of magnetic flux density non-linearly while the maximum is kept. The analysis helped to determine parameters of grooves dimensions which ensure high differences between min. and max. magnetic flux density, that means low ratio $R: i=3 ; w=6 \mathrm{~mm} ; h=3 \mathrm{~mm}$. These dimensions were used for the manufacture of a new magnetic circuit for the micro-fluidic system. The comparison of FEM model simulation results and the measurement of the B-x dependency is shown in Fig. $3 \mathrm{~b}$ for the current in the coil $\mathrm{I}=2 \mathrm{~A}$, orange and yellow curves for grooved pole pieces and blue curves for solid version of pole pieces. This figure also shows the measured $\mathrm{B}-\mathrm{x}$ curves for the current in the coil $\mathrm{I}=1 \mathrm{~A}$ and $\mathrm{I}=3 \mathrm{~A}$.
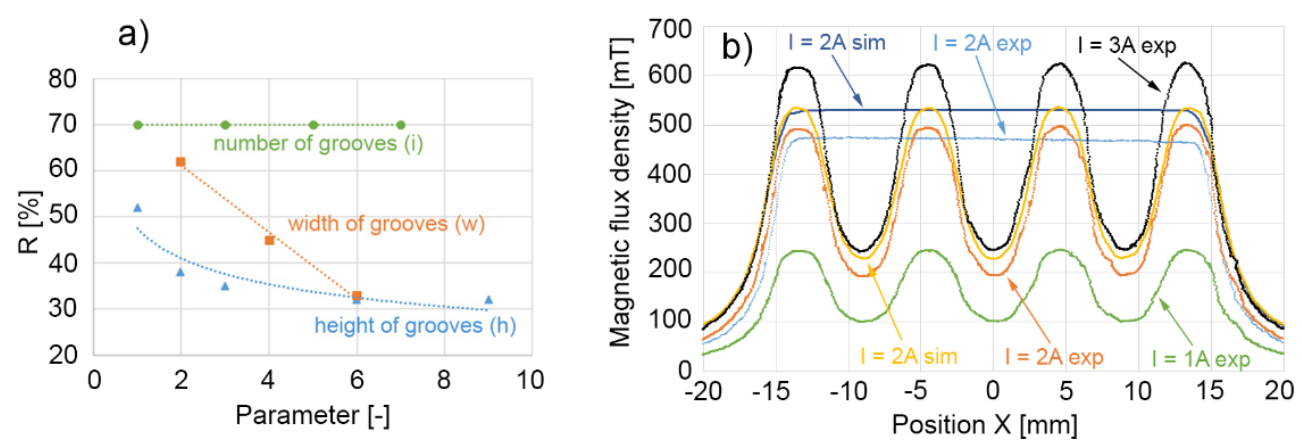

Fig. 3: Influence of grooves geometry to the ratio $R$ a), simulated and measured $B-x$ dependencies $b$ ). 


\subsection{Flow test through the chip of micro-fluidic system}

Two tests with the same methodology were carried out for both versions of magnetic circuit. The first flow of buffer through the chip causes that some amount of particles was wash out. This amount was higher for the solid version of magnetic circuit than for the grooved, compare the color of fluid in the test tubes marked as "2.5 A (first flow)" in Fig. 4. Another flows with lower currents in the coil did not caused any washout for the current higher than $1.25 \mathrm{~A}$ (for solid pole pieces) and $0.75 \mathrm{~A}$ (for grooved pole pieces of magnetic circuit). The test tubes with the washout samples for currents higher than $1.25 \mathrm{~A}$ were transparent. Therefore, they are not shown in the figure bellow. After the last flow of the buffer with the current in the coil $0.5 \mathrm{~A}$, the rest of particles in the chip was much higher for the grooved version of pole pieces than for the solid one.

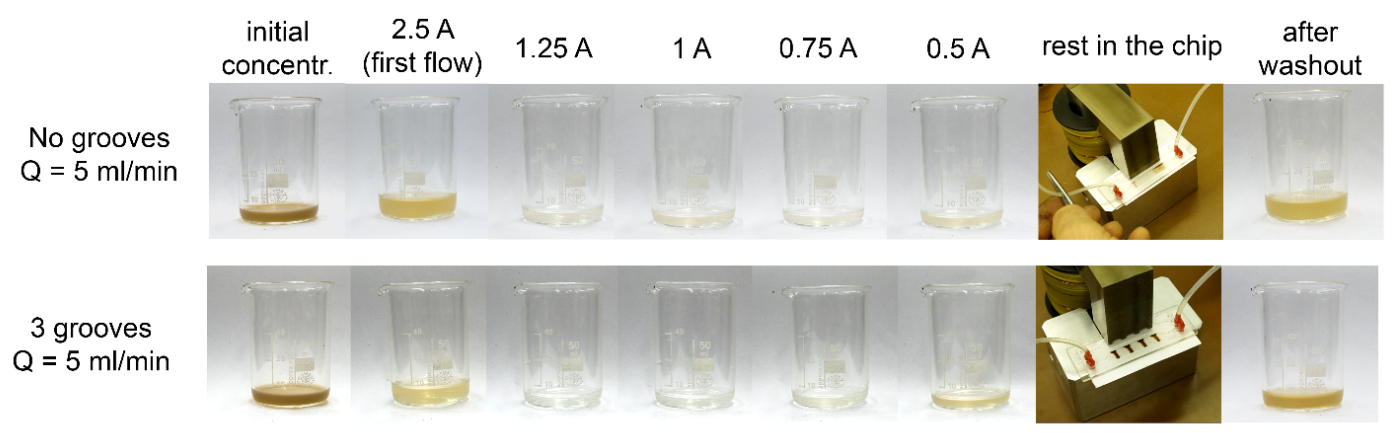

Fig. 4: The flow test of the micro-fluidic system.

\section{Conclusions}

Micro-fluidic system with an electromagnet was designed, manufactured and tested in this study. Two versions of pole pieces of the magnetic circuit were compared by magnetic flux density which is achieved in the gap for the same current in the coil and also by amount of particles which is captured in the chip for the same flow rate. The comparison shows that the grooved pole pieces of magnetic circuit have a positive impact to the total amount of particles which can be captured in the chip of microfluidic system. However, the results should be quantified, which is our next task. The initial concentration and volume of the input suspension has to be exactly kept for all tests as well as the amount of the buffer which is added into the input chamber between the current changes or before washout. However, the hypothesis that the grooved pole pieces of magnetic circuit of the micro-fluidic system should increase the amount of captured particles was confirmed by this study.

\section{Acknowledgement}

This experiment could be provided thanks to the kind sponsorship of various grants and numerous agencies. We would like to thank explicitly to the GAČR 17-10660J, GAČR 17-26162S and FSI-S-174428 .

\section{References}

Kubík, M., Macháček, O., Strecker, Z., Roupec, J. and Mazůrek, I. (2017) Design and testing of magnetorheological valve with fast force response time and great dynamic force range. Smart Materials and Structures, 26(4), 1-10.

Kuzhir, P., Magnet, C. Ezzaier, H., Zubarev, A. and Bossis, G. (2017) Magnetic filtration of phase separating ferrofluids. Journal of Magnetism and Magnetic Materials. 431, pp. 84-90.

Manz, A., Graber, H. and Widmer, H. M. (1990), Miniaturized total chemical analysis systém. Sens Actuators, B Chem, 1 (1-6) pp. 244-248.

Peng, H., Chen, B., He, M., Zhang Y. and Hu, B. (2011) Magnetic quantitative immunoanalysis of carcinoembryonic antigen by ICP-MS with mercury labels. Journal of Analytical Atomic Spectrometry. 26, 6, pp. 1217-1223.

Svobodová, Z., Krulišová, P., Černá, M., Jankovičová, B. and Bílková, Z.. (2015) On-Chip Elisa on Magnetic Particles: Isolation and Detection of Specific Antibodies from Serum. In: NANOCON 2015. Ostrava, Czech Republic: TANGER, pp. 477-482.

Zou, JB. and Lu, Y. (1992) Numerical Calculations for Ferrofluid Seals. IEEE Transactions on Magnetics 28, 4, pp. $3367-3371$. 